\title{
Penetration depth of photons in biological tissues from hyperspectral imaging in shortwave infrared in transmission and reflection geometries
}

Hairong Zhang

Daniel Salo

David M. Kim

Sergey Komarov

Yuan-Chuan Tai

Mikhail Y. Berezin 


\title{
Penetration depth of photons in biological tissues from hyperspectral imaging in shortwave infrared in transmission and reflection geometries
}

\author{
Hairong Zhang, Daniel Salo, David M. Kim, Sergey Komarov, Yuan-Chuan Tai, and Mikhail Y. Berezin* \\ Washington University School of Medicine, Department of Radiology, St. Louis, Missouri, United States
}

\begin{abstract}
Measurement of photon penetration in biological tissues is a central theme in optical imaging. A great number of endogenous tissue factors such as absorption, scattering, and anisotropy affect the path of photons in tissue, making it difficult to predict the penetration depth at different wavelengths. Traditional studies evaluating photon penetration at different wavelengths are focused on tissue spectroscopy that does not take into account the heterogeneity within the sample. This is especially critical in shortwave infrared where the individual vibration-based absorption properties of the tissue molecules are affected by nearby tissue components. We have explored the depth penetration in biological tissues from 900 to $1650 \mathrm{~nm}$ using Monte-Carlo simulation and a hyperspectral imaging system with Michelson spatial contrast as a metric of light penetration. Chromatic aberration-free hyperspectral images in transmission and reflection geometries were collected with a spectral resolution of $5.27 \mathrm{~nm}$ and a total acquisition time of $3 \mathrm{~min}$. Relatively short recording time minimized artifacts from sample drying. Results from both transmission and reflection geometries consistently revealed that the highest spatial contrast in the wavelength range for deep tissue lies within 1300 to $1375 \mathrm{~nm}$; however, in heavily pigmented tissue such as the liver, the range 1550 to $1600 \mathrm{~nm}$ is also prominent. ๑ 2016 Society of Photo-Optical Instrumentation Engineers (SPIE) [DOI: 10.1117/1.JBO.21.12.126006]
\end{abstract}

Keywords: shortwave infrared; extended NIR; deep tissue imaging; hyperspectral imaging; Michelson contrast; Monte-Carlo simulation.

Paper 160534PRR received Aug. 3, 2016; accepted for publication Nov. 11, 2016; published online Dec. 8, 2016.

\section{Introduction}

Achieving deep photon penetration is one of the main objectives for in vivo optical imaging and treatment with light-based therapies. The use of visible light is convenient but only feasible for skin and other directly accessible tissues (i.e., esophagus and colon $^{1}$ ) and is not suitable for deep tissue penetration due to the presence of strong absorption from endogenous chromophores (blood) ${ }^{2}$ and scattering from tissue components. Near-infrared (NIR) light is used to alleviate this scattering effect and is more suitable for deep tissue samples. Two optical windows exist within the NIR range: the first window ranges from 650 to $950 \mathrm{~nm}$ and is known as the traditional NIR or optical window $\mathrm{I}$, and the second window ranges from 950 to $2200 \mathrm{~nm}$ and is referred to as shortwave infrared (SWIR), extended NIR, or optical windows II to IV., ${ }^{3,4}$ While photon penetration in the traditional NIR range is well studied ${ }^{5-7}$ due to the wide availability of silicon-type detectors, light sources, and optical elements, the field of SWIR is relatively less explored and demands different types of instrumentation. ${ }^{8,9}$ Recent rapid developments in SWIR optics, and especially efficient InGaAs sensors between 900 and $1650 \mathrm{~nm}$ and SWIR lasers, have made this spectral range attractive for a variety of deep tissue therapeutic applications ${ }^{10}$ and deep tissue imaging. ${ }^{11,12}$

Recent studies have confirmed the feasibility of deep tissue imaging beyond $1000 \mathrm{~nm}^{11}$ with different contrast agents that emit in this wavelength such as quantum dots, ${ }^{13,14}$ carbon

*Address all correspondence to: Mikhail Y. Berezin, E-mail: berezinm@mir. wustl.edu nanotubes, ${ }^{15-17}$ and other emitters, including small molecules. ${ }^{18-20}$ A persistent challenge in SWIR imaging is identifying a set of wavelengths where tissue has the highest transparency. The answer for this question would allow the development of deeper imaging techniques with optimized light penetration and encourage the development of contrast agents that are optically active at the specifically identified (absorption and emission) wavelengths. The prediction of transparency at different wavelengths is complicated due to the contributions of several parameters, including Mie scattering, ${ }^{7}$ variations in absorption in accordance to the Beer-Lambert law, and anisotropy of tissue components. ${ }^{21}$ This complexity aggregates the demand for the experimental evidence of tissue transparency and the wavelength-depth relationship in SWIR.

Traditional studies evaluating photon penetration at different wavelengths have mostly focused on tissue spectroscopy that does not take into account the heterogeneity within the sample. Addressing heterogeneity is especially critical in SWIR where the individual vibration-based absorption properties of the tissue molecules such as water and lipids are affected by ions and nearby tissue components. ${ }^{22}$

We have previously demonstrated with the use of tissuemimicking phantoms (intralipid/India ink) that the wavelength-depth relationship can be evaluated via hyperspectral approaches using quantitative measurements of spatial Michelson contrast as a function of wavelength and depth. ${ }^{23}$ We also demonstrated that at low thicknesses of the phantom a reasonable contrast of the objects can be achieved at any wavelength between

$1083-3668 / 2016 / \$ 25.00$ @ 2016 SPIE 
700 and $1400 \mathrm{~nm}$ and between 1500 and $1600 \mathrm{~nm}$ in a transmission geometry. ${ }^{23}$ Such contrast can be achieved mostly between 1300 and $1350 \mathrm{~nm}$ at larger thicknesses. This previous study relied on a point-to-point scanning process that was inherently slow (up to $30 \mathrm{~min}$ for a scan), which prevented the investigation of a freshly excised, unfixed biological tissue. In this study, we have modified the hyperspectral imaging (HSI) system to enhance image acquisition speed. With the new system, we were able to investigate the tissue in 3 min or less, thus preventing imaging from many artifacts such as drying while maintaining high spatial and spectral resolution.

The goal of this work was to identify the wavelengths in SWIR at which the tissue has the highest transparency; therefore, we selected Michelson spatial contrast as a metric of light penetration. First, we designed and calibrated an HSI microscope to generate images free of chromatic aberrations. Second, we optimized the imaging conditions to record the data within 3 min to minimize the destruction of the biological tissue. We then investigated contrast as a function of the wavelength in phantoms and several types of tissue in transmission and reflection geometry. We also performed simulation studies to validate our experimental data.

\section{Materials and Methods}

\subsection{Instrument Design}

The design of the HSI system shown in Fig. 1 featured an SWIR sensitive two-dimensional (2-D) charge-coupled device (CCD) InGaAs camera NIRvana 640 (Princeton Instruments) for image acquisition mounted on the top of an Olympus BX51 microscope equipped with a triaxis PC-controlled stage (Marzhauser Wetzlar). A standard 100-W halogen microscope lamp (Olympus) with the IR filter removed was placed in front of the entrance imaging spectrograph iHR320 (Horiba Inc.) as the light source. Continuous broadband light passed through a 600 -grove $/ \mathrm{mm}$, blazed at $1000 \mathrm{~nm}$ grating, which dispersed light from 900 to $1650 \mathrm{~nm}$ into narrow bands. Narrow-band light was further delivered to the sample through a liquid light guide (Newport Corp.) with high transmission in the 420 to $2000 \mathrm{~nm}$ range. The design also included a calibration setup composed from a xenon lamp, double grating monochromator, liquid nitrogen-cooled InGaAs diode array CCD camera Symphony II, and a cuvette holder with a standard made from Spectralon with high reflection (all components from Horiba Inc.).

Both transmission and reflection geometries were used for data acquisition. In the transmission geometry, the light delivered by the light guide passed through a microscope condenser and onto the sample. In the reflection geometry, the light delivered by the light guide projected onto the sample passed through a beamsplitter and a chromatic aberration-free $15 \times$ reflective objective (Beck) attached to the microscope through a set of adapters (Thorlabs) (Fig. 1). The transmitted or reflected light was collected with the same objective and channeled into the 2-D camera. Integration time, slit size, and wavelength increments were set in the FluorEssence software (Horiba). Image acquisition was collected through the LightField software (Princeton Instruments) and was synchronized with the wavelength selection.

\subsection{Objective Selection for Chromatic Aberration-Free Imaging}

The imaging system was corrected for chromatic aberrations, which is a known problem in HSI. ${ }^{24}$ In the visible and NIR ranges, chromatically corrected objectives such as apochromats can mitigate the image distortion caused by chromatic aberrations. These objectives accommodate low dispersion glass elements to neutralize the difference in refracting indices at variable wavelengths; however, apochromatic objectives fully corrected in SWIR are not commercially available. One noticeable exception exists and is collectively known as a reflective objective. These types of objectives, first developed by Schwarzchild (circa 1904), ${ }^{25}$ are based on a mirror system with no glass elements. Due to the all-reflecting optics, the objectives are chromatic aberration-free in the very broad spectral range from UV

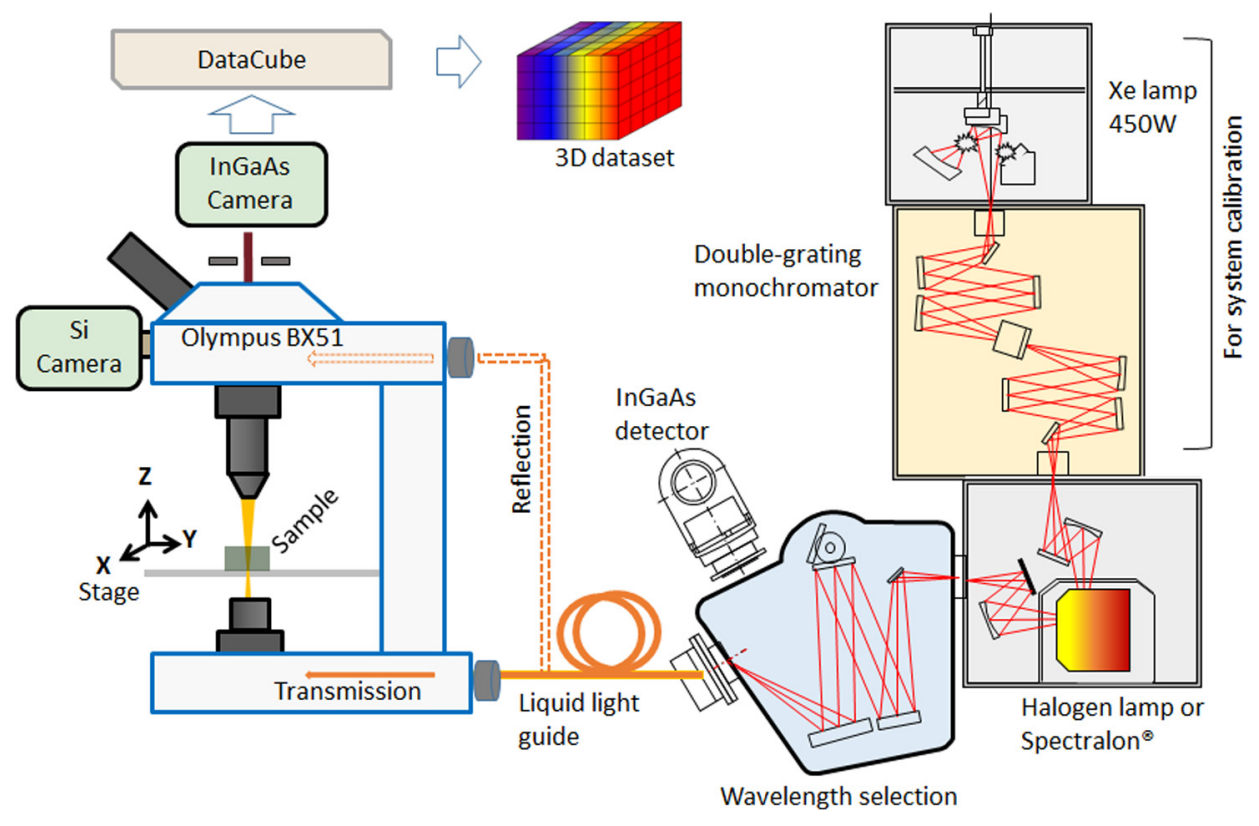

Fig. 1 Schematic of the HSI system for contrast measurement in phantoms in the spectral range 900 to $1650 \mathrm{~nm}$. 


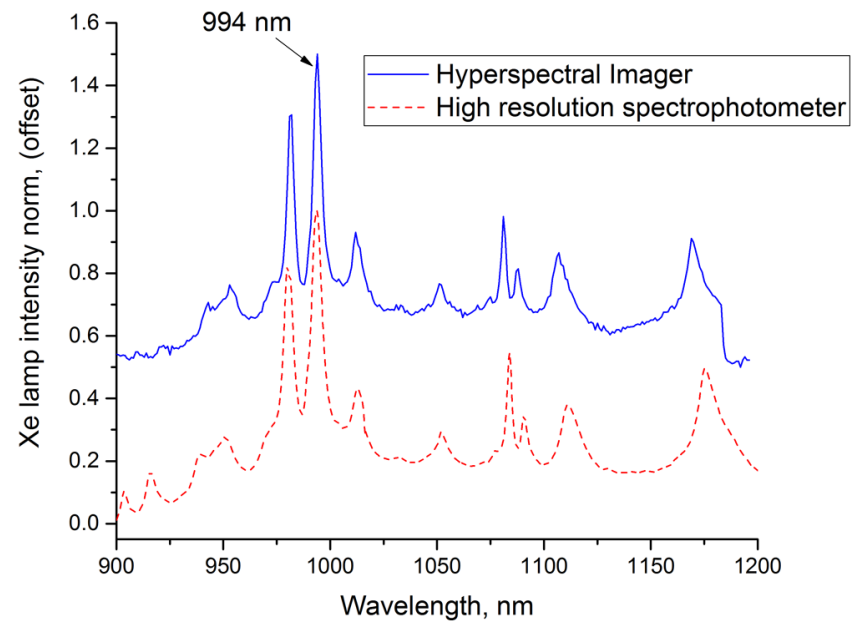

Fig. 2 Spectral profiles of the xenon lamp as measured by the highresolution calibrated spectrophotometer and $\mathrm{HSI}$. Similar appearance of the spectra collected from the imager and from the high-resolution spectrophotometer indicated spectrally calibrated HSI system and also demonstrate sufficient spectral resolution. All peaks are within $\pm 2 \mathrm{~nm}$ of each other.

$(<300 \mathrm{~nm}) \mathrm{nm}$ to IR $(>10 \mu \mathrm{m})$. In the current work, we used a $15 \times$ reflective objective (working distance $23.5 \mathrm{~mm}$ and $\mathrm{NA}=0.28$, Beck) for all image acquisitions.

\subsection{Spectral Calibration and Spectral Resolution}

Spectral calibration was performed to ensure the correct spectral response of the HSI system. First, the detector Symphony II was calibrated using a neodymium doped glass that emits at $1052 \mathrm{~nm}$ when excited at $575 \mathrm{~nm}$. Second, a xenon lamp emission was recorded using the calibrated Symphony II detector with a high spectral resolution (increment $0.25 \mathrm{~nm}$, integration time $5 \mathrm{~ms}$, emission slit $1 \mathrm{~nm}$ ) to set the position of a reference peak at 994 nm (Fig. 2). Finally, the HSI was spectrally calibrated with a 994-nm emission line from the xenon lamp (increment $1.0 \mathrm{~nm}$, emission slit $1 \mathrm{~nm}$ ).

Spectral resolution has an important effect on HSI performance, and determining optimal resolution is critical in HSI measurements. Low spectral resolution ( $>20 \mathrm{~nm}$ bandwidth) limits the ability of the method to resolve closely spaced spectral features, while high spectral resolution $(<3 \mathrm{~nm})$ requires significant increases in the integration time, which lowers output. Spectral resolution in the presented HSI system was adjusted by the width of the slits in the monochromator and the selection of the wavelength increment. The influence of both parameters on the broadening of the signal measured as full-width-at-halfmaximum (FWHM) from a narrow linewidth $1064 \mathrm{~nm}$ laser is illustrated in Figs. 3 and 4. The results demonstrate a strong outcome of the slit width on FWHM, with fourfold FWHM increase from $1 \mathrm{~nm}$ slit to $8 \mathrm{~nm}$ (Fig. 3). The effect of the wavelength increment was somewhat less significant (1.5-fold increase FWHM from $1 \mathrm{~nm}$ increment to $4 \mathrm{~nm}$, Fig. 4). The optimized image acquisition parameters were set to $5 \mathrm{~nm}$ slit and $5.27 \mathrm{~nm}$ increment.

\subsection{Sample Preparation}

The phantom's stock solution was composed of $18.75 \mathrm{~mL}$ intralipid (20\% fat emulsion, Fresenius Kabi), $0.62 \mathrm{~mL}$ India ink (2\% in water, Speedball), and $300 \mathrm{~mL}$ water. ${ }^{26}$ Total amount of lipid was $1.1 \mathrm{w} . \%$, which is similar to the amount typically seen in freshly excised chicken breast $(0.98 \pm 0.23 \%)$. ${ }^{27}$ The absorption coefficients for individual components such as water and lipids in SWIR have been measured in our previous work. ${ }^{3}$ Based on the literature data, the $\mu_{\mathrm{a}}$ values of intralipid phantoms are within 0.15 to 0.17 at $1100 \mathrm{~nm}$ and depend strongly on the wavelength following the shape of the absorption spectra. ${ }^{28}$ The values $\mu_{\mathrm{a}}^{\prime}$ decrease monotonically on average from $4 \mathrm{~cm}^{-1}$ at $1100 \mathrm{~nm}$ to $1 \mathrm{~cm}^{-1}$ at $1700 \mathrm{~nm}$.

The phantom assembly utilized a grid made from a stainless steel card (Fotofab Inc.) perforated with evenly spaced holes of about $230 \mu \mathrm{m}$ diameter attached beneath a plastic polystyrene
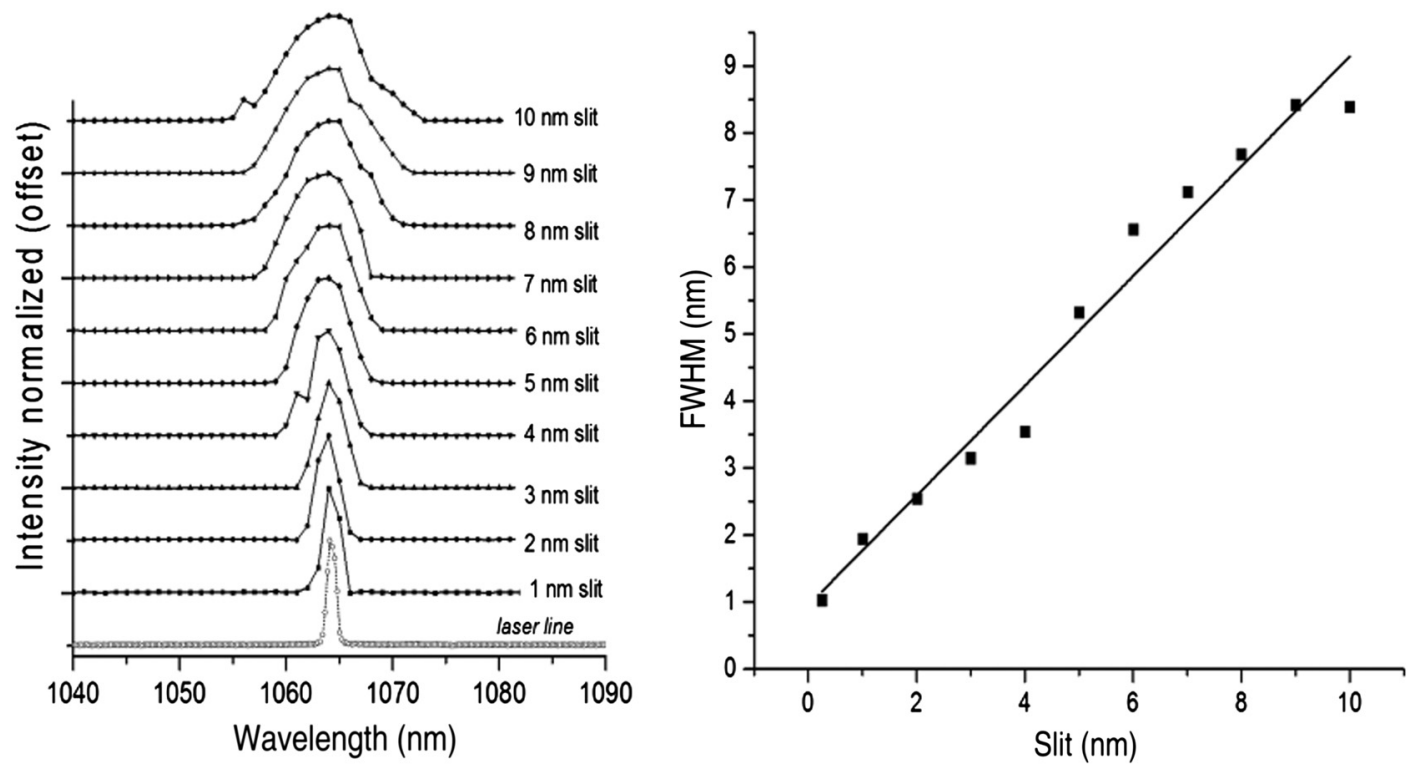

Fig. 3 Effect of slits on the HSI spectral resolution. FWHM of the recorded laser line at $1064 \mathrm{~nm}$ shows linear broadening of the signal at larger slits. Laser line (true line) was recorded with a high spectral resolution spectrophotometer. The spectra are normalized and plotted with an offset. 

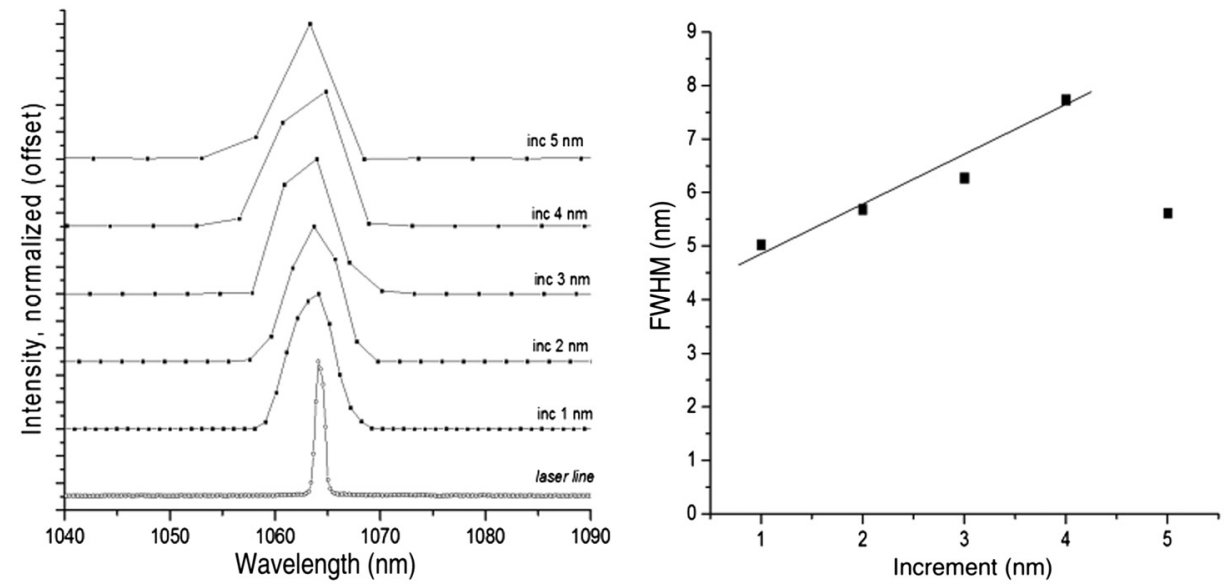

Fig. 4 Effect of the wavelength increment on the HSI spectral resolution. FWHM of the recorded laser line at $1064 \mathrm{~nm}$ shows broadening of the signal at larger increments. The outlier at $5 \mathrm{~nm}$ was due to low sampling rate below Nyquist value. The spectra are normalized and plotted with an offset.
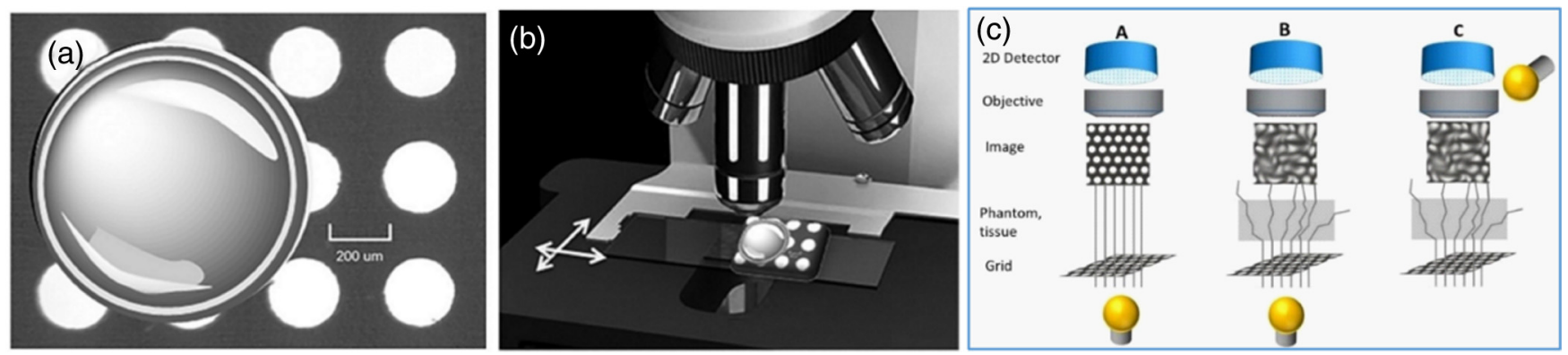

Fig. 5 (a) Phantom/tissue setup (closed-up view), (b) phantom/tissue placement, (c) principle of phantom and tissue setup for contrast measurements with hyperspectral imaging showing transmission $(A$ and $B)$ and reflection $(C)$ mode.

petri-dish $(35 \mathrm{~mm} \times 10 \mathrm{~mm})$ that was filled with the phantom stock solution (Fig. 5). The stock ranged from about 2 to $7 \mathrm{~mL}$, corresponding to depths of 3.0 to $6.5 \mathrm{~mm}$ in the middle of the petri-dish, where the meniscus effect is minimal.

\subsection{Tissue Preparation}

Fresh chicken tissues including thigh, breast, and liver were obtained from a local supermarket (Whole Foods, St. Louis, Missouri), cut in small pieces and placed between two 25-mmdiameter cover glasses (Deckglaser) to ensure uniform distribution of thickness and prevent from drying. The thickness of the tissue was determined by measuring the distance between the two cover glasses using a digital caliper. The tissue was then placed on a $230-\mu \mathrm{m}$-diameter grid for contrast measurement.

\subsection{Data Acquisition}

The focus was adjusted manually prior to image sequence acquisition using a PC-controlled stage (Marzhauser Wetzlar) at $1300 \mathrm{~nm}$ to achieve the best visual contrast of the grid. A number of 180 collected frames corresponded to the spectral resolution of $5.27 \mathrm{~nm}$. Dispersed light was coupled with a light guide with the distal end connected to either a transmission or a reflection port of the microscope. The power of the lamp, the slit width on the spectrograph, and the integration time of the camera for each image captured were optimized to achieve an adequate signal strength $(>1000$ counts, with the noise level $<100$ counts). Using these optimized conditions, the integration time was set to $1 \mathrm{~s}$, which brought the total time of recording to $3 \mathrm{~min}$.

\subsection{Data Processing}

The set of collected frames were analyzed with ImageJ using the OpenSPE extension for direct export of frames. Regions of interest (ROIs) in the light and dark areas in the image (holes or the metal parts) were selected as shown in Fig. 6. Approximate maximum and minimum light intensities were obtained for each frame (wavelength) by averaging the light intensities in the ROIs selected with the plot $Z$-axis profile function. The Michelson contras $^{29}$ was then calculated according

$\operatorname{contrast}(\lambda)=\left(I_{\text {light }}-I_{\text {dark }}\right) /\left(I_{\text {light }}+I_{\text {dark }}\right)$,

where $I_{\text {light }}$ and $I_{\text {dark }}$ correspond to the average light and dark intensities of the selected areas. A contrast value of 0 corresponds to no contrast and a contrast value of 1 corresponds to maximal contrast.

\section{Results and Discussion}

HSI-SWIR is a relatively established technique. Historically, satellite-based remote sensing was the first large-scale application of HSI-SWIR. ${ }^{30}$ In the last decade, with the advances in 

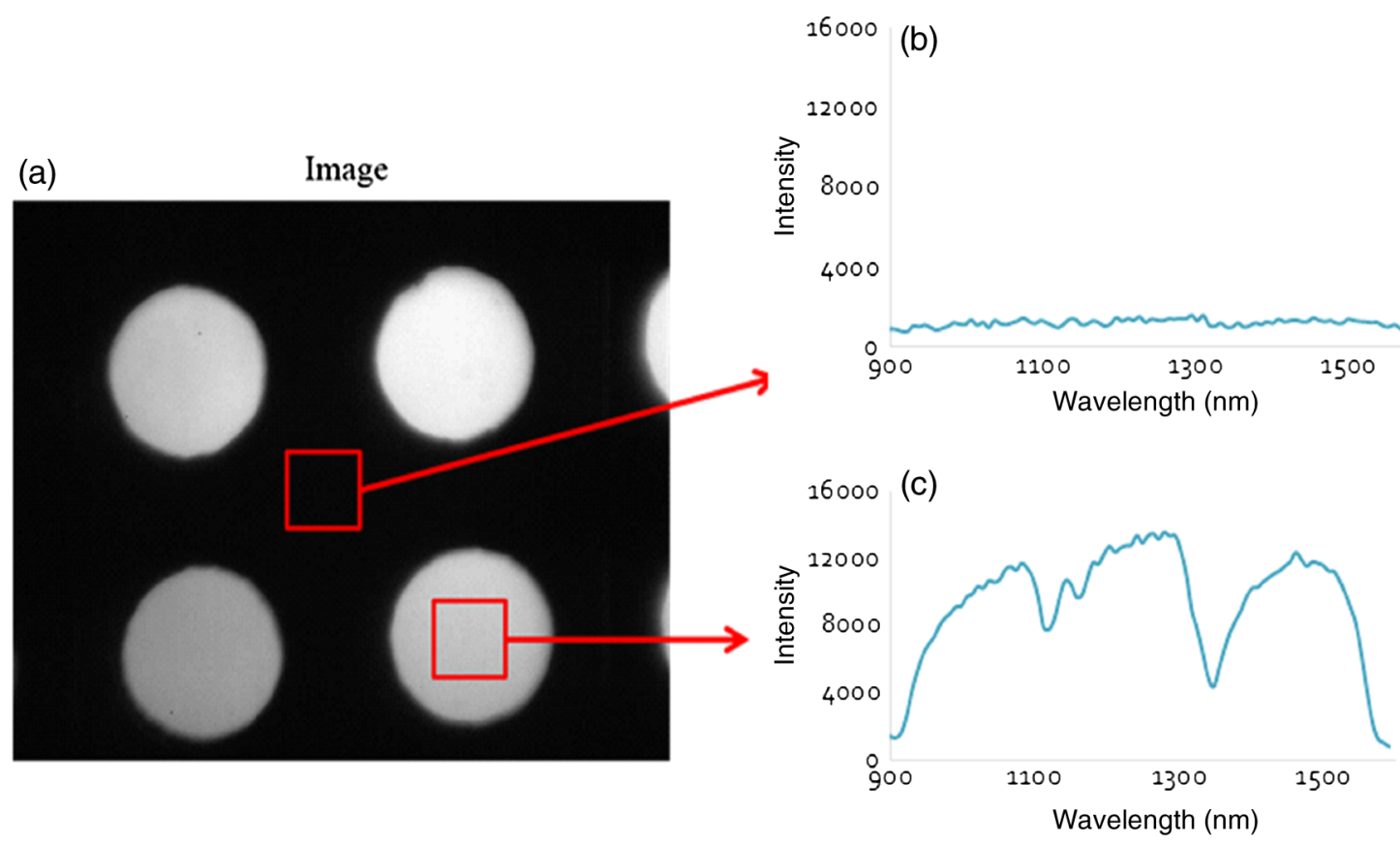

Fig. 6 Calculation of the Michelson contrast from the datacube and ROls corresponding to the light and dark areas in the image. (a) Representative frame of a grid covered with a tissue-mimicking phantom. (b) Average intensity of the dark ROI over the spectral range from 900 to $1650 \mathrm{~nm}$. (c) Average intensity of the ROI inside the hole over the spectral range from 900 to $1650 \mathrm{~nm}$.

InGaAs technology, the applications of HSI-SWIR have been significantly broadened and currently cover a variety of research fields such as security, ${ }^{4}$ plant science, ${ }^{31,32}$ agriculture, ${ }^{33}$ and food analysis. ${ }^{34}$ The latter includes characterization of the food products, ${ }^{35}$ detection of contaminants, ${ }^{36}$ and quality control. ${ }^{37}$ Given the better penetration of SWIR photons in scattering media, SWIR-HSI has also been recognized as a viable tool to monitor objects through thick smoke, ${ }^{38}$ detect hidden damages in fruits, ${ }^{39}$ and diagnose and demarcate deep bruises in humans. ${ }^{40}$ In this work, we employ HSI-SWIR to measure the wavelengthdependent photon penetration in phantoms and different biological tissues.

Our method of measuring penetration depth was based on the quantitative evaluation of the spatial Michelson contrast introduced in our earlier publications. ${ }^{23,41}$ In this method, a sample of interest was placed on top of a grid, as shown schematically in Fig. 5, and the contrast was measured (as shown in the previous section and Fig. 6). Image contrast was independent of wavelength for a blank sample provided that the system was corrected for chromatic aberrations and the wavelength of detection was within detector sensitivity. The blank sample refers to the measurements from the Fotofab card without any of the intralipid/ India stock solution. The contrast of the blank grid sample under transmission geometry in Fig. 7(a) shows this independence by exhibiting a steady contrast within the 0.88 to 0.91 range (1.0 under ideal conditions) from 935 to $1625 \mathrm{~nm}$. The contrast substantially decreases at the margins due to the drop in sensitivity of the InGaAs sensor at these wavelengths, which makes the measurements below 535 and above $1625 \mathrm{~nm}$ no longer reliable. The value of the measured contrast was a little lower (less than 10\%) than the expected value of 1.0 under ideal conditions.

The data for the transmission and reflection modes presented in Figs. 7(a) and 7(b) show general trends between contrast and wavelength consistent for both geometries. Significantly lower depth penetration in the reflection geometry when compared to the transmission geometry was due in part to an average doubling in path length within the tissue. The increased photon path length of the reflection geometry leads to relatively more absorption events, which results in a lower $I_{\text {light }}$. Fresnel reflection also contributed to the lower depth penetration of the reflection geometry because of the involvement of a greater number of surfaces with different refractive indices. ${ }^{42,43}$ The lowest contrast within the studied spectral range was at 1400 to $1500 \mathrm{~nm}$ due to the strong water absorption at these wavelengths. The contrast at other wavelengths depended on sample thickness. At lower thickness, the highest contrasts were centered at two spectral bands: 1300 to $1375 \mathrm{~nm}$ and 1575 to $1625 \mathrm{~nm}$. As the thickness of the phantoms increased, the contrast at the $1300 \mathrm{~nm}$ band became much more prominent than at $1600 \mathrm{~nm}$ [Fig. 7(c)].

These results reflect a compromise between the absorption properties of the chromophores and scattering. While the absorption coefficients vary greatly over the SWIR spectrum, the scattering coefficient of tissue decreases monotonically according to the Mie theory. ${ }^{44}$ At $1600 \mathrm{~nm}$, the absorption coefficient of water, the dominant chromophore, is an order of magnitude higher than that at $1300 \mathrm{~nm} 5.7 \mathrm{~cm}^{-1}$ versus $77.5 \mathrm{~cm}^{-1} ;{ }^{45}$ however, the scattering at $1600 \mathrm{~nm}$ is expected to be substantially lower than at $1300 \mathrm{~nm}$. Under ideal Rayleigh scattering conditions, scattering is proportional to $\lambda^{-4} \cdot{ }^{33}$ With particles size similar to the wavelength of the incident electromagnetic radiation (Mie conditions), the scattering in SWIR is less wavelength-dependent and proportional from $\lambda^{-1.5}$ to $\lambda^{-0.6}$ depending on the type of tissue and often method of measurements. ${ }^{8,46}$ Based on this evaluation, thick tissue is expected to have the highest transparency at 1300 and $1600 \mathrm{~nm}$, and this result is confirmed by our experimental data [Fig. 7(c)] and simulation (see Figs. 9 and 10). 

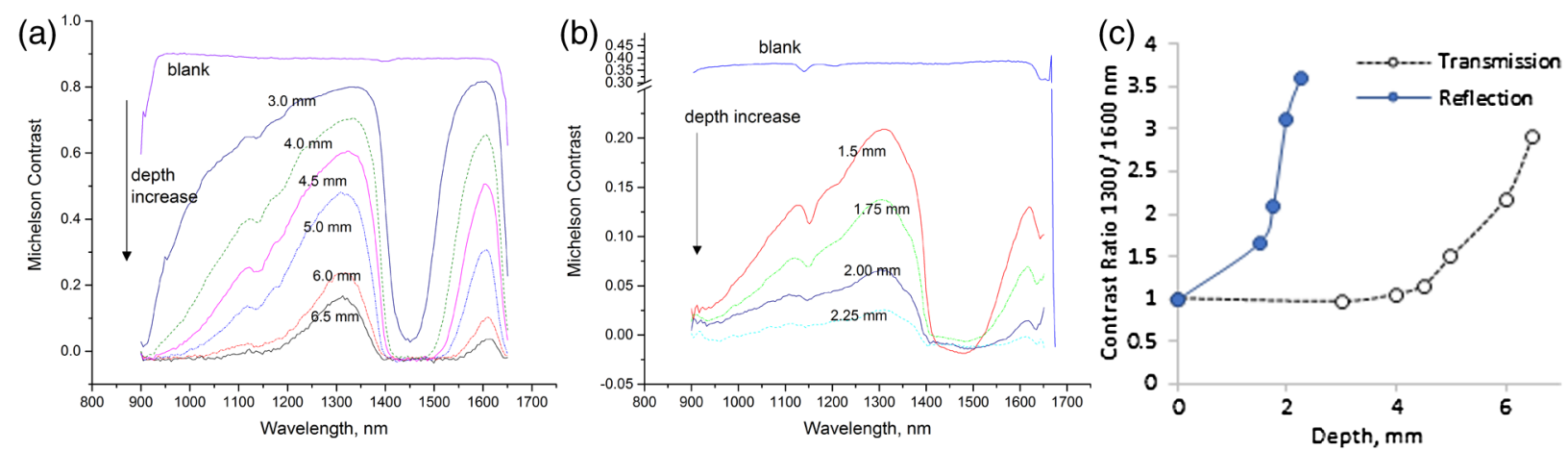

Fig. 7 Michelson contrast values as a function of wavelength from 900 to 1650 at different depth in intralipid/India ink phantoms from 0 to $6.5 \mathrm{~mm}$ for intralipid/India ink phantoms with $230-\mu \mathrm{m}$-diameter grid. (a) Transmission geometry. (b) Reflection geometry. (c) Ratio of two Michelson contrast values (1300 to $1600 \mathrm{~nm}$ ) at different depths.

Figure 8 summarizes the contrast values for representative tissues such as chicken thigh, breast, and liver in the transmission and reflection geometries at different thickness. Different organs have a distinct chemical composition (fat and proteins),

(a) 0.95 Thigh Transmission

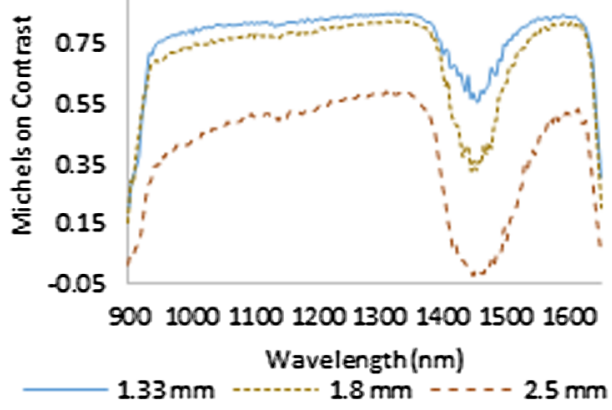

(c)
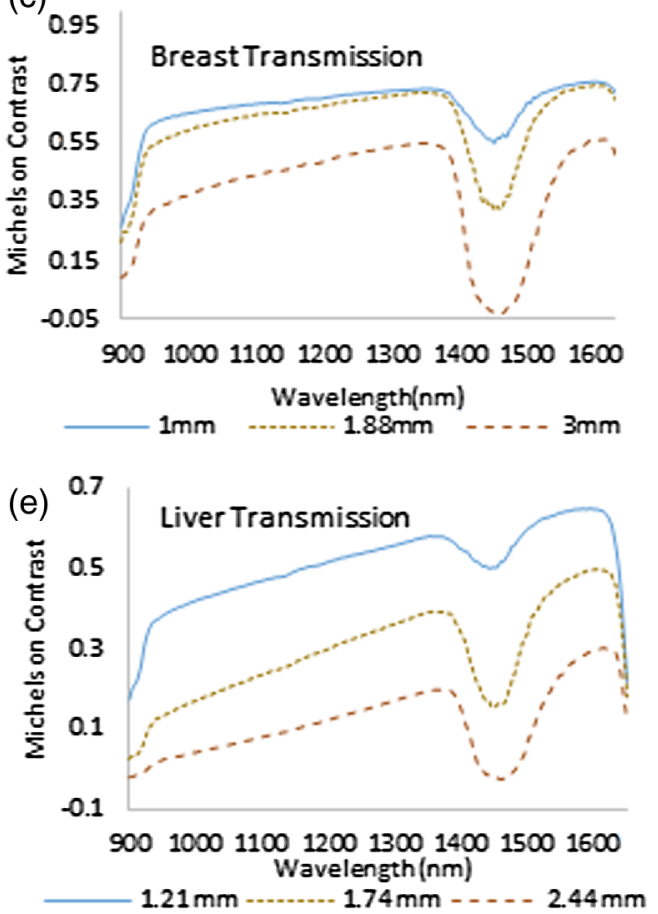

presence of pigments (i.e., hemoglobin), and connective tissue that contributes to scattering.

All tissues presented a loss of contrast at around 1400 to $1450 \mathrm{~nm}$ due to the high level of water in the tissue $(>70 \%$

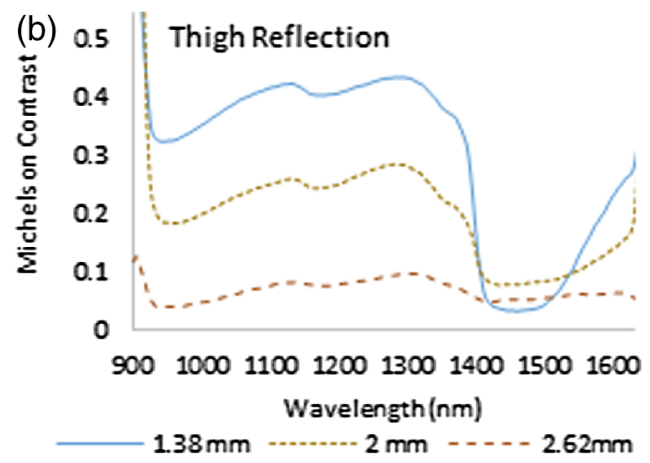

(d)
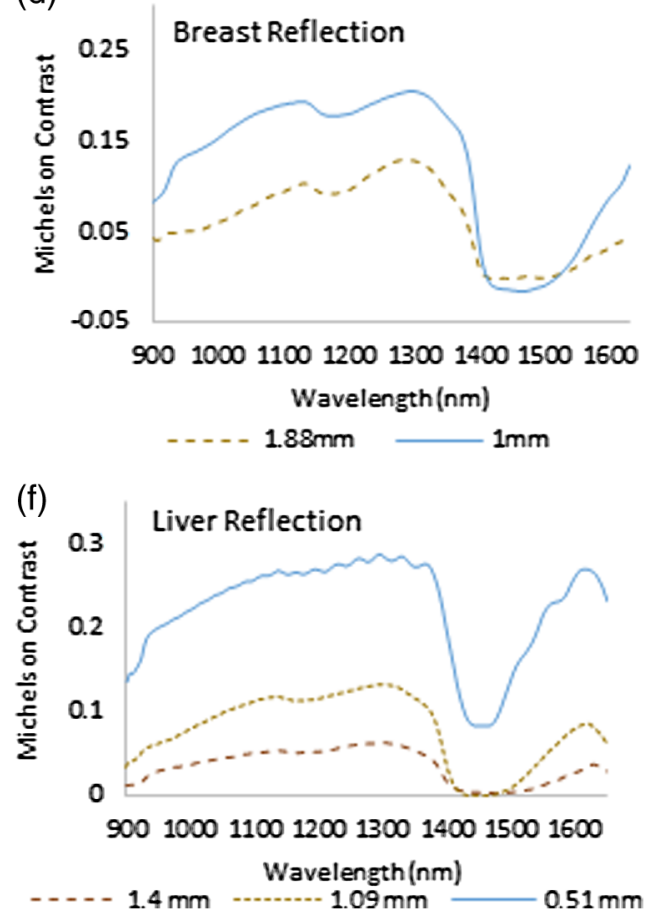

Fig. 8 Contrast data at different type of tissues and depth across wavelengths from 900 to $1650 \mathrm{~nm}$. (a) Chicken thigh transmission, (b) chicken thigh reflection, (c) chicken breast transmission, (d) chicken breast reflection, (e) chicken liver transmission, and (f) chicken liver reflection. $230-\mu \mathrm{m}$-diameter grid. 

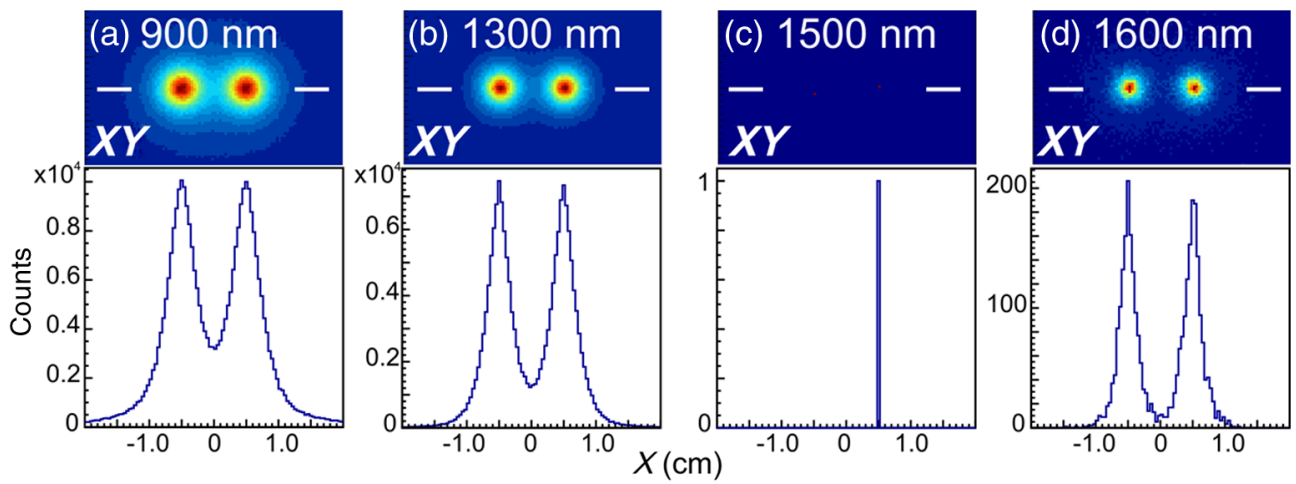

Fig. 9 Representative Monte-Carlo simulation of two parallel photon beam distributions in biological tissue in transmission geometry. (a) $900 \mathrm{~nm}: \mu_{\mathrm{a}}=0.07 \mathrm{~cm}^{-1}, \mu_{\mathrm{s}}^{\prime}=1.3 \mathrm{~cm}^{-1}$; (b) $1300 \mathrm{~nm}$ : $\mu_{\mathrm{a}}=$ $0.8 \mathrm{~cm}^{-1}, \mu_{\mathrm{s}}^{\prime}=1.07 \mathrm{~cm}^{-1}$; (c) $1500 \mathrm{~nm}: \mu_{\mathrm{a}}=13 \mathrm{~cm}^{-1}, \mu_{\mathrm{s}}^{\prime}=0.95 \mathrm{~cm}^{-1}$; (d) $1600 \mathrm{~nm}: \mu_{\mathrm{a}}=5 \mathrm{~cm}^{-1}$, $\mu_{\mathrm{s}}^{\prime}=0.85 \mathrm{~cm}^{-1}$.

in most of the soft tissues ${ }^{47}$ ), which correlated with the trend observed with the intralipid/India ink phantoms. The contrast steadily increased with the wavelength, providing the largest contrast at 1300 to 1375 and 1575 to $1625 \mathrm{~nm}$, which also correlated with the trend observed with the phantoms. These two bands showed similar contrast in the transmission geometry, which is important for light-based therapies. The contrast at 1300 to $1375 \mathrm{~nm}$ was consistently higher and particularly evident at larger depths in the reflection geometry, which is more practical for medical imaging. Freshly excised chicken tissue retains most of its interstitial fluid from the lymphatic system $\left(>70 \%\right.$ moisture $\left.{ }^{27}\right)$ but, in general, contains a reduced amount of blood. We expect that the presence of blood will increase the absorption coefficient due to the increased level of oxygenated hemoglobin that is still significantly absorbing in this spectral range (although less than in the NIR and visible). ${ }^{3,48}$

To explain the results, we performed a Monte-Carlo simulation. Because of a lack of optical data in the studied tissues (chicken breast, thigh, and liver), Monte-Carlo simulation was performed using literature data on absorption and reduced scattering coefficients in rat skin. ${ }^{49}$ Scattering was modeled using the standard Henyey-Greenstein phase function. ${ }^{50}$ In this simulation, two parallel beams of photons at different wavelengths (each beam $=1 \times 10^{7}$ photons) entered the tissue perpendicular to the surface. Reflection/transmission coefficients were calculated using the known Fresnel equation for nonpolarized photons through infinite XY surface of 10-mm thickness. The results of such simulations in the transmission geometry are

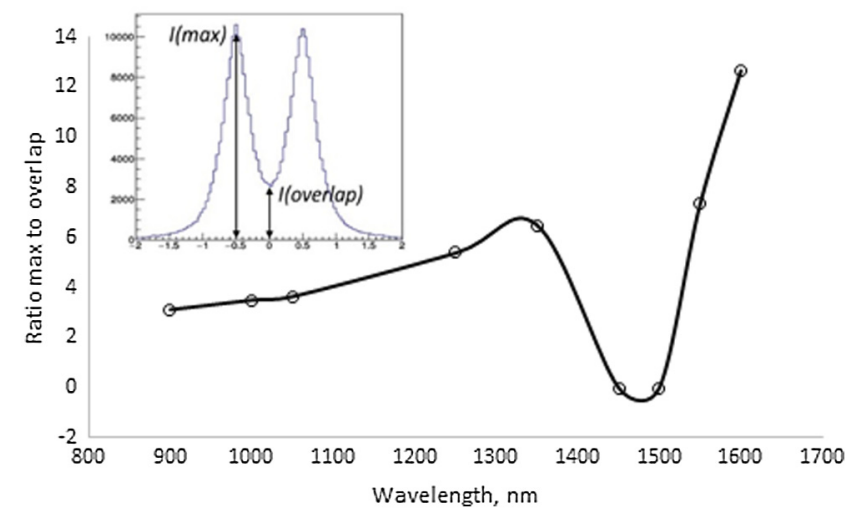

Fig. 10 Calculated resolution of two-peak histograms: $R=I_{\max } / I_{\text {overlap }}$. shown in Fig. 9. The resolution between two peaks was calculated as $R=I_{\max } / I_{\text {overlap }}$ and presented in Fig. 10 as a function of wavelength. The trend of resolution versus wavelength was quite similar to what we measured experimentally in the transmission geometry with maximums at 1350 and $1550 \mathrm{~nm}$. Beams were set $10 \mathrm{~mm}$ apart, $1 \times 10^{7}$ photons in each beam. Thickness of the tissue was set to $10 \mathrm{~mm}$. For simplification, the values of the refractive index $n$ and anisotropy $g$ were considered to be independent from the wavelength in the 900 to $1600 \mathrm{~nm}$ range and equal to $n=1.4$ (typical values of $n$ for the tissue from 1.35 to $1.41^{8}$ and reviewed in Ref. 51), and anisotropy factor $g=0.9$ (typical values 0.80 to $0.95^{52}$ ). The upper panel shows the distribution of the photon from the bottom view (entrance) and top view. The lower panel shows the histogram of photons number from the middle position between the two beams.

Analysis of the tissue-based data revealed the difference between the types of the tissue. While thigh and breast showed similar contrast apparently due to the similarity in their tissue composition, the liver presented a significantly lower contrast (Fig. 11). This difference became especially noticeable at larger thickness. The liver is highly vascularized compared to the thigh and the breast tissues and is therefore richer in red cells and hemoglobin. Hemoglobin is strongly absorbing in the visible but also absorbing at longer wavelengths with the absorption coefficient at $1300 \mathrm{~nm}$ comparable to that of water. ${ }^{3}$ Thus, despite the known low scattering coefficient of the liver compared to other tissues, ${ }^{6}$ the liver is substantially less transparent in the SWIR than other soft tissues such as breast and thigh.

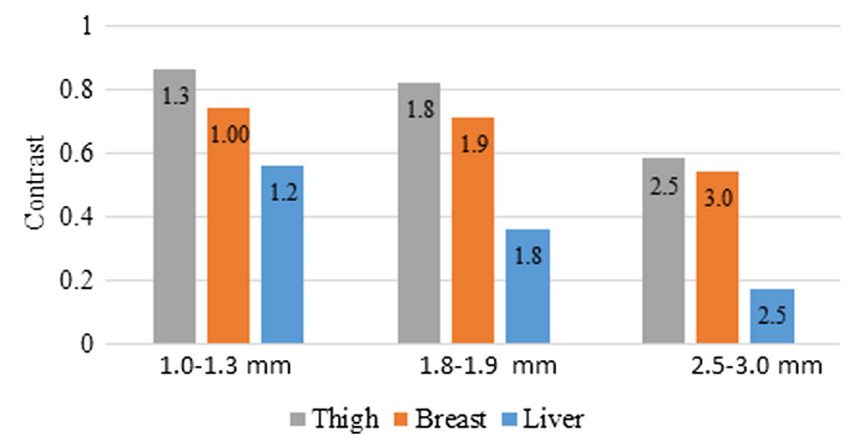

Fig. 11 Michelson contrast of biological tissues at different depth at $1300 \mathrm{~nm}$. Values in the columns show the actual thickness of the tissue in $\mathrm{mm}$. 
There are a growing number of publications that explore the SWIR range as a new optical window II. ${ }^{13,53,54}$ This optical range has been explored quite recently for the diagnostics of middle ear pathologies ${ }^{55}$ and arthritis. ${ }^{56}$ In this regard, our study confirms that light penetration in certain spectral ranges within the SWIR range is higher than in the more traditional NIR range. In this work, we have experimentally demonstrated that the highest transparency lies in a narrow range of 1300 to $1375 \mathrm{~nm}$ and in some cases (i.e., transmission geometry) in the range of 1575 to $1625 \mathrm{~nm}$.

Since tissue lipids have a distinct absorption profile, HSISWIR can be applied to identify a lipid-based plaque in the in vivo and in vitro models of atherosclerosis. ${ }^{57,58}$ As the detection of the coronary plaque is often identified with an optical fiberscope catheter, the outcome of the study will depend not only on the depth penetration but also on the contrast between the plaque and the blood vessel. This contrast can be significantly improved using our HSI-SWIR imaging approach as we have presented elsewhere. ${ }^{59}$

The presented contrast versus wavelength data obtained from the HSI approach contain the combined information about scattering, absorption, and other optical parameters. In general, the observed trend follows the light penetration patterns reported in the literature. ${ }^{8}$ In the NIR $(<900 \mathrm{~nm})$ range, the scattering in general dominates the absorption $\mu_{\mathrm{s}}^{\prime}>\mu_{\mathrm{a}}$, providing relatively low contrast. At longer wavelengths $(<1000 \mathrm{~nm})$, where the scattering values are comparable to those of absorption ( $\mu_{\mathrm{s}}^{\prime} \approx \mu_{\mathrm{a}}$ ), the contrast increases with the decrease of scattering. Higher values of absorption $\left(\mu_{\mathrm{a}} \gg \mu_{\mathrm{s}}^{\prime}\right)$ even in combination with relatively low scattering, as observed in the 1400- to $1500-\mathrm{nm}$ range, block the light penetration and consequently lead to a weak contrast. Such variability in light penetration in SWIR has been observed in a number of studies. $8,28,49,60$

\section{Conclusions and Future Work}

Using HSI, we investigated the transparency of the tissue at different wavelengths in SWIR. We focused on a spectral range from 900 to $1650 \mathrm{~nm}$ measuring the depth penetration in biological tissues using a Michelson spatial contrast as a metric of light penetration. Chromatic aberration-free hyperspectral images in transmission and reflection geometries were collected with a spectral resolution of $5.27 \mathrm{~nm}$ and a total acquisition time of $3 \mathrm{~min}$. Relatively short recording time minimized artifacts from sample drying. Results from both transmission and reflection geometries in phantoms and biological tissues consistently revealed the highest spatial contrast within 1300 to $1375 \mathrm{~nm}$ in deep tissues. The most critical parameters affecting depth penetration in soft tissue were water and hemoglobin absorptions. In heavily pigmented tissue such as liver, the depth penetration was significantly lower than for other studied tissues such as the breast and the thigh. The presented data opens up the possibility for the rational design of fluorescent probes and light-based therapies for deeper penetration, which could have potential applications in clinical settings. We have recently identified QDs with the high quantum yield in the desired optical range at about $1300 \mathrm{~nm}^{61}$

Our finding suggests that 1300 to $1375 \mathrm{~nm}$ is the best optical band for deep tissue imaging within the tested range; however, the poor sensitivity of the commercial InGaAs detector beyond $1650 \mathrm{~nm}$ did not allow us to measure the contrast at longer wavelengths. It has been recently reported that light penetration is increasing up to $1800 \mathrm{~nm}^{4,11}$ The measurement of the
Michelson contrast in these longer wavelength would require a different type of detector with sufficient sensitivity.

As part of our long-term interest in SWIR imaging, we are currently developing a computational model of SWIR light penetration in a variety of biological media. This theoretical model requires reliable quantitative values of absorption and reduced scattering coefficients in SWIR, as well as other wavelengthrelated parameters, such as anisotropy and refractive index. Although these parameters are available for some tissues, such as skin, they cannot be directly applied to the studied type of tissues. We are currently developing a method to extract lighttissue penetration parameters in SWIR using the generated contrast-wavelength dependence. A related work is currently being conducted in our lab and will be reported in a separate paper.

\section{Disclosures}

The authors have nothing to disclose.

\section{Acknowledgments}

Dr. Berezin reports grants from NIH, grants from NSF, during the conduct of the study: the NCI/NIH CA198419, the NSF Research Infrastructure Improvement Award \# IIA-1355406, and the Washington University Optical Spectroscopy Core Facility (NIH 1S10RR031621-01).

\section{References}

1. A. N. G. Bashkatov et al., "Optical properties of human colon tissues in the 350-2500 nm spectral range," Quant. Electron. 44(8), 779-784 (2014).

2. M. Friebel et al., "Influence of oxygen saturation on the optical scattering properties of human red blood cells in the spectral range 250 to 2 , 000 nm," J. Biomed. Opt. 14(3), 034001 (2009).

3. Q. Cao et al., "Multispectral imaging in the extended near-infrared window based on endogenous chromophores," J. Biomed. Opt. 18(10), 101318 (2013).

4. L. Shi et al., "Transmission in near-infrared optical windows for deep brain imaging," J. Biophotonics 9(1-2), 38-43 (2016).

5. J. V. Frangioni, "In vivo near-infrared fluorescence imaging," Curr. Opin. Chem. Biol. 7(5), 626-634 (2003).

6. S. L. Jacques, "Optical properties of biological tissues: a review," Phys. Med. Biol. 58(11), R37-R61 (2013).

7. V. Ntziachristos et al., "Looking and listening to light: the evolution of whole-body photonic imaging," Nat. Biotechnol. 23(3), 313-320 (2005).

8. T. L. Troy and S. N. Thennadil, "Optical properties of human skin in the near infrared wavelength range of 1000 to $2200 \mathrm{~nm}$," J. Biomed. Opt. 6(2), 167-176 (2001).

9. A. N. Bashkatov et al., "Optical properties of human skin, subcutaneous and mucous tissues in the wavelength range from 400 to $2000 \mathrm{~nm}$," J. Phys. D Appl. Phys. 38, 2543-2555 (2005).

10. A. Mester, "Laser biostimulation," Photomed. Laser Surg. 31(6), 237239 (2013).

11. L. A. Sordillo et al., "Deep optical imaging of tissue using the second and third near-infrared spectral windows," J. Biomed. Opt. 19(5), 056004 (2014).

12. X. Gao et al., "In vivo cancer targeting and imaging with semiconductor quantum dots," Nat. Biotechnol. 22(8), 969-976 (2004).

13. C. Li et al., "In vivo real-time visualization of tissue blood flow and angiogenesis using $\mathrm{Ag}_{2} \mathrm{~S}$ quantum dots in the NIR-II window," Biomaterials 35(1), 393-400 (2014).

14. Y. Kong et al., "Highly fluorescent ribonuclease-a-encapsulated lead sulfide quantum dots for ultrasensitive fluorescence in vivo imaging in the second near-infrared window," Chem. Mater. 28(9), 3041-3050 (2016).

15. N. Won et al., "Imaging depths of near-infrared quantum dots in first and second optical windows," Mol. Imaging 11(4), 338-352 (2012). 
16. Y. Jung et al., "Quantum dot imaging in the second near-infrared optical window: studies on reflectance fluorescence imaging depths by effective fluence rate and multiple image acquisition," J. Biomed. Opt. 20(4), 046012 (2015).

17. K. Welsher, S. P. Sherlock, and H. Dai, "Deep-tissue anatomical imaging of mice using carbon nanotube fluorophores in the second nearinfrared window," Proc. Natl. Acad. Sci. U. S. A. 108(22), 8943-8948 (2011).

18. D. R. Klaus et al., "1D polymeric platinum cyanoximate: a strategy toward luminescence in the near-infrared region beyond $1000 \mathrm{~nm}$," Inorg. Chem. 54(4), 1890-1900 (2015).

19. Z. Tao et al., "Biological imaging using nanoparticles of small organic molecules with fluorescence emission at wavelengths longer than 1000 nm," Angew. Chem. Int. Ed. Engl. 52(49), 13002-13006 (2013).

20. A. L. Antaris et al., "A small-molecule dye for NIR-II imaging," Nat. Mater. 15(2), 235-242 (2016).

21. A. M. Smith, M. C. Mancini, and S. Nie, "Bioimaging: second window for in vivo imaging," Nat. Nanotechnol. 4(11), 710-711 (2009).

22. R. Rottgers, D. McKee, and C. Utschig, "Temperature and salinity correction coefficients for light absorption by water in the visible to infrared spectral region," Opt. Express 22(21), 25093-25108 (2014).

23. D. Salo et al., "Multispectral measurement of contrast in tissue-mimicking phantoms in near-infrared spectral range of 650 to $1600 \mathrm{~nm}$," J. Biomed. Opt. 19(8), 086008 (2014).

24. J. Amako, K. Nagasaka, and N. Kazuhiro, "Chromatic-distortion compensation in splitting and focusing of femtosecond pulses by use of a pair of diffractive optical elements," Opt. Lett. 27(11), 969-971 (2002).

25. D. S. Grey, "A new series of microscope objectives: II. Preliminary investigation of catadioptric Schwarzschild systems," J. Opt. Soc. Am. 39(9), 723-728 (1949).

26. A. Q. Bauer et al., "Quantitative photoacoustic imaging: correcting for heterogeneous light fluence distributions using diffuse optical tomography," J. Biomed. Opt. 16(9), 096016 (2011).

27. S. Mudalal et al., "Quantity and functionality of protein fractions in chicken breast fillets affected by white striping," Poultr. Sci. 93(8), 2108-2116 (2014).

28. I. Bargigia et al., "Time-resolved diffuse optical spectroscopy up to $1700 \mathrm{~nm}$ by means of a time-gated InGaAs/InP single-photon avalanche diode," Appl. Spectrosc. 66(8), 944-950 (2012).

29. G. E. Legge et al., "Psychophysics of reading. XI. Comparing color contrast and luminance contrast," J. Opt. Soc. Am. A 7(10), 20022010 (1990).

30. A. F. H. Goetz, "Three decades of hyperspectral remote sensing of the Earth: a personal view," Remote Sens. Environ. 113(Suppl. 1), S5-S16 (2009).

31. D. M. Kim et al., "Highly sensitive image-derived indices of waterstressed plants using hyperspectral imaging in SWIR and histogram analysis," Sci. Rep. 5, 15919 (2015).

32. J. Xing et al., "Comparison of short-wavelength infrared (SWIR) hyperspectral imaging system with an FT-NIR spectrophotometer for predicting alpha-amylase activities in individual Canadian western red spring (CWRS) wheat kernels," Biosyst. Eng. 108(4), 303-310 (2011).

33. C. Riccioli et al., "Pixel selection for near-infrared chemical imaging (NIR-CI) discrimination between fish and terrestrial animal species in animal protein by-product meals," Appl. Spectrosc. 65(7), 771-781 (2011).

34. M. S. Kim et al., "Visible to SWIR hyperspectral imaging for produce safety and quality evaluation," Sens. Instrum. Food Qual. Saf. 5(5), 155-164 (2011).

35. J. Burger and P. Geladi, "Hyperspectral NIR imaging for calibration and prediction: a comparison between image and spectrometer data for studying organic and biological samples," Analyst 131(10), 1152-1160 (2006).

36. X. Fu et al., "Investigation of NIR hyperspectral imaging for discriminating melamine in milk powder," Proc. SPIE 8721, 87210F (2013).

37. H. Huang, L. Liu, and M. Ngadi, "Recent developments in hyperspectral imaging for assessment of food quality and safety," Sensors 14(4), 7248-7276 (2014).

38. M. K. Griffin et al., "Characterization and delineation of plumes, clouds and fires in hyperspectral images," in IEEE Proc. Int. Geoscience and Remote Sensing Symp. (IGARSS), pp. 809-812 (2000).
39. J. C. Keresztes, M. Goodarzi, and W. Saeys, "Real-time pixel based early apple bruise detection using short wave infrared hyperspectral imaging in combination with calibration and glare correction techniques," Food Control 66, 215-226 (2016).

40. L. L. Randeberg and J. Hernandez-Palacios, "Hyperspectral imaging of bruises in the SWIR spectral region," Proc. SPIE 8207, 82070N (2012).

41. H. Zhang et al., "Penetration depth in tissue-mimicking phantoms from hyperspectral imaging in SWIR in transmission and reflection geometry," Proc. SPIE 9703, 970311 (2016).

42. C. Chen et al., "A primary method for determination of optical parameters of turbid samples and application to intralipid between 550 and 1630 nm," Opt. Express 14(16), 7420-7435 (2006).

43. L. A. Sordillo et al., "Imaging of tissue using a NIR supercontinuum laser light source with wavelengths in the second and third NIR optical windows," Proc. SPIE 9319, 93191Y (2015).

44. J. L. Sandell and T. C. Zhu, "A review of in-vivo optical properties of human tissues and its impact on PDT," J. Biophotonics 4(11-12), 773787 (2011).

45. R. Röttgers et al., "Pure water spectral absorbtion, scattering, and real part of refractive index model," in ESA Algorithm Technical Basis Document, pp. 1-18, ESA STSE WaterRadiance Project, HelmholtzZentrum Geesthacht, Geesthacht, Germany (2010).

46. R. Nachabé et al., "Estimation of biological chromophores using diffuse optical spectroscopy: benefit of extending the UV-VIS wavelength range to include 1000 to $1600 \mathrm{~nm}, "$ Biomed. Opt. Express 1(5), 1432-1442 (2010).

47. H. H. Mitchell et al., "The chemical composition of the adult human body and its bearing on the biochemistry of growth," J. Biol. Chem. 158(3), 625-637 (1945).

48. E. Salomatina and A. Yaroslavsky, "Evaluation of the in vivo and ex vivo optical properties in a mouse ear model," Phys. Med. Biol. 53(11), 2797-2807 (2008).

49. R. H. Wilson et al., "Quantitative short-wave infrared multispectral imaging of in vivo tissue optical properties," J. Biomed. Opt. 19(8), 086011 (2014).

50. L. G. Henyey and J. L. Greenstein, "Diffuse radiation in the galaxy," Astrophys. J. 93, 70-83 (1941).

51. P. Giannios et al., "Visible to near-infrared refractive properties of freshly-excised human-liver tissues: marking hepatic malignancies," Sci. Rep. 6, 27910 (2016).

52. A. Roggan et al., "Medizinphysik: diagnostik und therapie mit dem laser," Phys. J. 55(3), 25-30 (1999).

53. G. Hong et al., "Near-infrared II fluorescence for imaging hindlimb vessel regeneration with dynamic tissue perfusion measurement," Circ. Cardiovasc. Imaging 7(3), 517-525 (2014).

54. E. C. Ximendes et al., "LaF3 core/shell nanoparticles for subcutaneous heating and thermal sensing in the second biological-window," Appl. Phys. Lett. 108(25), 253103 (2016).

55. J. A. Carr et al., "Using the shortwave infrared to image middle ear pathologies," Proc. Natl. Acad. Sci. U. S. A. 113(36), 9989-9994 (2016).

56. L. A. Paluchowski et al., "Identification of inflammation sites in arthritic joints using hyperspectral imaging," Proc. SPIE 8947, 89470H (2014).

57. J. Wang et al., "Near-infrared spectroscopic characterization of human advanced atherosclerotic plaques," J. Am. Coll. Cardiol. 39(8), 13051313 (2002).

58. T. Hasegawa, I. Sogawa, and H. Suganuma, "A near infrared angioscope visualizing lipid within arterial vessel wall based on multi-spectral image in $1.7 \mu \mathrm{m}$ wavelength band," Proc. SPIE 8575, 857506 (2013).

59. D. Salo et al., "Extended near-infrared: a new window on in vivo bioimaging," BioOptics World 7(1), (2014).

60. X. Ma et al., "Bulk optical parameters of porcine skin dermis at eight wavelengths from 325 to 1557 nm," Opt. Lett. 30(4), 412-414 (2005).

61. A. P. Aydt et al., "Synthesis and spectroscopic evaluation of PbS quantum dots emitting at $1300 \mathrm{~nm}$ for optimized imaging in optical window II," Proc. SPIE 9723, $97230 \mathrm{Z}$ (2016).

Hairong Zhang received her BS degree from Washington University in 2016. She worked in Mikhail Berezin's lab at the Department of Radiology at Washington University School of Medicine from 2013 to 2016. Her primary research interest included developing hyperspectral optical imaging systems as well as image processing algorithms. She is currently a PhD candidate at Columbia University. 
Daniel Salo is a graduate student at Duke University. He spent his summer as an amgen scholar in the laboratory of Mikhail Berezin and has since received his MS in biomedical engineering from Duke University. He has an interest in computational modeling and its application to cutting-edge healthcare technologies.

David M. Kim received his BS degree from Washington University in 2016. He worked in Mikhail Berezin's lab at the Department of Radiology at Washington University School of Medicine from 2012 to 2015.

Sergey Komarov, $\mathrm{PhD}$, is a senior scientist at Washington University School of Medicine in St. Louis. His research interests includes nuclear and plasma physics, positron emission tomography, image reconstruction, Cherenkov luminescence imaging, gamma spectroscopy and the spectroscopy of highly ionized atoms.
Yuan-Chuan Tai is the associate professor of radiology and biomedical engineering at Washington University School of Medicine in St. Louis. He is focusing on positron-emission technology for highresolution preclinical and human imaging applications.

Mikhail Y. Berezin, PhD, is the assistant professor at Washington University School of Medicine in St. Louis. His research interests include optical spectroscopy, hyperspectral optical imaging, and design of contrast agents in near- and shortwave infrared for tissue imaging and medical diagnostics. He is the author of more than 60 peer-reviewed papers, books and book chapters in this field. He is a senior member of SPIE. 